\title{
Etiología microbiana de la conjuntivitis neonatal en un hospital de la ciudad de Medellín
}

\section{Microbial etiology of neonatal conjunctivitis in a hospital in the city of Medellín}

\author{
Martha L. Gaviria-Bravo ${ }^{1,2,3 *}$, Yenny Llano-Naranjo ${ }^{1}$ y Oscar A. Villada-Ochoa ${ }^{4}$ \\ ${ }^{1}$ Departamento de Oftalmología, Universidad de Antioquia, Medellín; ²División de Oftalmología Pediátrica y Estrabismo, Fundación Oftalmológica \\ de Santander, Santander; ${ }^{3}$ Departamento de Oftalmopediatría, Hospital Universitario San Vicente Fundación, Medellín; ${ }^{4}$ Dirección de Investigaciones, \\ Hospital Universitario San Vicente Fundación, Medellín. Colombia
}

\section{Resumen}

Objetivo: Describir la etiología microbiana, factores de riesgo asociados y sensibilidad antibiótica de la conjuntivitis neonatal en el Hospital Universitario San Vicente Fundación de Medellín en el periodo de 2012 a 2018. Metodología: Estudio descriptivo, ambispectivo, desde enero de 2012 a noviembre de 2018 en donde se revisaron las historias clínicas de pacientes con diagnóstico de conjuntivitis neonatal con resultados de cultivos positivos de secreción conjuntival en un hospital de tercer nivel en la ciudad de Medellín. Se estudiaron variables sociodemográficas de la madre y el neonato, factores de riesgo asociados, resultados de los aislamientos microbiológicos, terapia antibiótica tópica y sistémica y sensibilidad antibiótica. Resultados: Se identificaron un total de 69 neonatos, 65.22\% de sexo masculino, con una edad media de 15.7 \pm 8.3 días. Los principales agentes etiológicos encontrados fueron Staphylococcus epidermidis (20 pacientes, 28.9\%), Staphylococcus aureus (12 pacientes, 17.3\%) y Haemophilus influenzae (9 pacientes, $13.0 \%$ ). Con las pruebas realizadas no se obtuvo crecimiento para Neisseria gonorrhoeae, ni hubo resultado positivo para Chlamydia trachomatis. Los factores de riesgo maternos periparto encontrados con mayor frecuencia fueron: la ruptura prematura de membranas ovulares $(n=16$, $21.33 \%)$, infección del tracto urinario $(n=7,10.1 \%)$, síndrome de parto pretérmino $(n=6,8.00 \%)$ y vulvovaginosis $(n=5$, 7.25\%). Para la familia de Staphylococcus se encontró resistencia a la oxacilina del $26 \%$ ( $n=18)$ y del $15 \%(n=11)$ a las tetraciclinas. Conclusión: El perfil epidemiológico de la conjuntivitis neonatal ha cambiado en la última década. Actualmente la causa más frecuente son agentes no gonocócicos. Se encontró un importante número de pacientes con Staphylococcus aureus meticilinorresistente.

Palabras clave: Conjuntivitis neonatal. Factores de riesgo. Cultivo. Bacterias.

\section{Abstract}

Objective: To describe the microbial etiology, maternal risk factors and antibiotic sensitivity of neonatal conjunctivitis at San Vicente Hospital Foundation in Medellín since 2012-2018. Methodology: A descriptive, ambispective trial was carried out between January 2012 and November 2018. We reviewed the medical records of patients diagnosed with neonatal bacterial 
conjunctivitis with positive cultures of conjunctival secretion. Sociodemographic variables of the mother and the neonate, associated risk factors, results of the microbiological isolations, topical and systemic antibiotic therapy and antibiotic sensitivity were recorded. Results: $A$ total of 69 newborns were identified, $65.22 \%$ were male, the average age was $15.7 \pm 8.3$ days. Major etiological agents identified were Staphylococcus epidermidis $(n=20,28.9 \%)$, Staphylococcus aureus $(n=12$, $17.3 \%$ ) and Haemophilus influenzae (9 patients, 13.0\%). There were no positive isolates for Chlamydia trachomatis or Neisseria gonorrhoeae. The most common maternal peripartum risk factors were premature rupture of the ovular membranes $(n=16,21.33 \%)$, urinary tract infection (7 cases, $10.1 \%)$, preterm birth syndrome $(n=6,8.00 \%)$ and bacterial vaginosis $(5$ cases, $7.25 \%)$. Resistance to oxacillin $26 \%(n=18)$ and tetracyclines $15 \%(n=11)$ was observed in patients with Staphylococcus infection. Conclusion: Over the years, the epidemiology of neonatal conjunctivitis has changed. Nowadays, gonococcal pathogens are not the most common cause of neonatal conjunctivitis. A significant number of patients with methicillin-resistant $S$. aureus were observed.

Key words: Neonatal conjunctivitis. Risk factors. Culture. Bacteria.

\section{Introducción}

La conjuntivitis neonatal (CN) es una infección aguda de la membrana conjuntival frecuente en la edad neonatal, que, de no tratarse, conlleva el riesgo de pérdida visual $^{1-3}$. La prevalencia oscila entre el 1.6 y el $11.3 \%{ }^{4}$, aumenta en países en desarrollo y es causa de ceguera en cerca de 10.000 neonatos por año. De ellos, la mitad en el continente africano ${ }^{2,5}$.

La causa de la CN puede ser de origen infeccioso 0 no infeccioso. Dentro del primer grupo, en el siglo XX, antes del uso generalizado de profilaxis ocular, la Chlamydia trachomatis y la Neisseria gonorrhoeae, microorganismos relacionados con infecciones de transmisión sexual, eran los principales agentes etiológicos ${ }^{6}$. Estudios publicados recientemente continúan reportando la $C$. trachomatis como causa importante de $\mathrm{CN}$ en algunos países en desarrollo ${ }^{7,8}$, pero en menor proporción comparado con tiempos pasados.

Actualmente, en diferentes estudios realizados sobre la epidemiología de la $\mathrm{CN}$, los agentes bacterianos de la flora de la piel y del ambiente (Staphylococcus sp.) es decir, bacterias no gonocócicas, son aislados con mayor frecuencia en cultivos de secreción conjuntival2,9,10.

Esta transición de la epidemiología de la $\mathrm{CN}$ obedece a la introducción de la profilaxis ocular en el recién nacido (RN), mejoras en la calidad de los programas de atención materna, del parto y tratamientos oportunos $^{11,12}$. En las regiones donde no se encuentren estas mejoras, la prevalencia por microorganismos de origen genitourinario continúa siendo alta ${ }^{3}$.

Hay pocos estudios sobre la etiología microbiana de la $\mathrm{CN}$ a nivel local, y los que hay fueron publicados hace más de una década ${ }^{13,14}$. Por estas razones, se desarrolló el presente trabajo con el objetivo de establecer los principales agentes causales, su sensibilidad antibiótica y posibles factores de riesgo asociados.

\section{Materiales y métodos}

Se realizó un estudio de tipo observacional, descriptivo, ambispectivo, en dos fases, en el total de pacientes con diagnóstico de $\mathrm{CN}$ atendidos en el Hospital Universitario de San Vicente Fundación, en Medellín, Colombia, entre enero de 2012 y diciembre de 2017 (fase retrospectiva) y entre enero y noviembre de 2018 (fase prospectiva). Los criterios de inclusión fueron: diagnóstico clínico de $\mathrm{CN}$, menores de 30 días de edad, con cultivos positivos de secreción conjuntival y con registro de los resultados de sensibilidad a los antibióticos disponibles para cada cultivo. Se excluyeron los pacientes con diagnóstico de dacrioestenosis, celulitis preseptal, o queratoconjuntivitis.

Se identificaron las siguientes variables sociodemográficas de la madre y el RN (edad, género, procedencia), de la madre (vía de parto, infecciones asociadas periparto, número de controles prenatales, complicaciones periparto no infecciosas) y del RN (cultivo para bacterias aerobias en agar sangre y agar chocolate, detección de antígeno para C. trachomatis y cultivo especial en ThayerMartin para N. gonorrheae, presencia de dacrioestenosis u otra enfermedad ocular asociada, enfermedad sistémica concomitante, factores de riesgo periparto en la madre, tipo de antibióticoterapia recibida tópica y sistémica en el neonato y sensibilidad antibiótica).

La información de los resultados de los cultivos de secreción conjuntival positivos fue proporcionada por el laboratorio clínico del Hospital Universitario de San Vicente Fundación. La información de las variables demográficas y clínicas se extrajeron de las historias clínicas electrónicas de los pacientes, las cuales reposan en el sistema operativo SAP del Hospital Universitario San Vicente Fundación.

Para el procesamiento de la información se elaboró una base de datos en Microsoft Excel Office $365^{\circledR}$ 
(Vermont, EE.UU) con las variables mencionadas. El análisis estadístico se llevó a cabo con el software R. Las variables cualitativas se describen según frecuencias absolutas y relativas para cada categoría. Las variables cuantitativas se seguían con una distribución normal empleando la prueba Kolmogorov Smirnov. Las variables que distribuyen normal se describen según su promedio y desviación estándar. Todos los investigadores participaron en la producción y aprobación del documento final. La investigación fue avalada por el Comité de Ética del Hospital Universitario de San Vicente de Fundación y de la Universidad de Antioquia.

\section{Resultados}

\section{Variables demográficas}

Entre enero de 2012 y noviembre de 2018 se revisaron 106 historias clínicas de neonatos con diagnóstico clínico de CN y cultivos positivos de secreción conjuntival. En 24 casos (22\%) se diagnosticó dacrioestenosis y en $3(2.8 \%)$ celulitis periorbitaria, por lo cual fueron excluidos del análisis. Hubo 10 pacientes que tenían una edad de más de 30 días y no cumplían con la definición de $\mathrm{CN}$, por lo que tampoco se incluyeron en el estudio. Finalmente se incluyeron en el análisis un total de 69 pacientes, 45 (65.2\%) eran del sexo masculino y $24(34.78 \%)$ del sexo femenino. Las características demográficas se resumen en la tabla 1.

El $75.3 \%(n=52)$ de los partos fueron por vía vaginal y el $24.6 \%(n=17)$ por cesárea. La mayoría de estos pacientes se diagnosticaron en el servicio de urgencias ( $n=41,59.2 \%$ ), unidad cuidados intensivos neonatales $(n=14,20.2 \%)$ y salas generales pediátricas $(n=11$, $15.9 \%$ ). Todos los pacientes recibieron manejo intrahospitalario.

Se evaluó la presencia de infección genitourinaria asociada al periodo periparto en las madres de los RN, y se encontró la infección del tracto urinario (ITU) en el 10.4\% $(n=7)$, seguida de la vulvovaginosis en el $7.25 \%(n=5)$ y tamizaje para $S$. agalactiae positivo en el $5.8 \%(n=4)$. En el $44.93 \%(n=31)$ de los casos no se reportó ninguna infección genitourinaria asociada al periodo periparto.

Se estudiaron las complicaciones periparto en las madres y su relación con los neonatos que desarrollaron $\mathrm{CN}$, y se determinó que la ruptura prematura de membranas ovulares (RPMO) $(n=16,21.3 \%)$, el síndrome de parto pretérmino (SPP) $(n=6,8 \%)$ y, en menor porcentaje, el trastorno hipertensivo asociado al embarazo (THAE) $(n=4,5.3 \%)$ fueron los factores más frecentes (Tabla 1).
Tabla 1. Variables demográficas $(\mathrm{n}=69)$

\begin{tabular}{|c|c|c|}
\hline Variable & n & $\%$ \\
\hline Edad neonatos (días) ${ }^{*}$ & \multicolumn{2}{|c|}{$15 \pm 8.3$} \\
\hline $\begin{array}{l}\text { Género neonato } \\
\text { Masculino } \\
\text { Femenino }\end{array}$ & $\begin{array}{l}45 \\
24\end{array}$ & $\begin{array}{l}65.2 \\
34.8\end{array}$ \\
\hline $\begin{array}{l}\text { Procedencia madre } \\
\text { Urbana } \\
\text { Rural } \\
\text { Sin registro }\end{array}$ & $\begin{array}{c}32 \\
35 \\
2\end{array}$ & $\begin{array}{r}46.4 \\
50.7 \\
2.9\end{array}$ \\
\hline Edad gestacional madre (semanas) & \multicolumn{2}{|c|}{$35 \pm 4.8$} \\
\hline Número controles prenatales* & \multicolumn{2}{|c|}{$5.3 \pm 3.2$} \\
\hline $\begin{array}{l}\text { Vía del parto } \\
\text { Vaginal } \\
\text { Cesárea }\end{array}$ & $\begin{array}{l}52 \\
17\end{array}$ & $\begin{array}{l}75.4 \\
24.6\end{array}$ \\
\hline $\begin{array}{l}\text { Servicio de atención y diagnóstico del } \\
\text { neonato } \\
\text { Urgencias de pediatría } \\
\text { Salas generales de hospitalización } \\
\text { UCIN } \\
\text { UCEN }\end{array}$ & $\begin{array}{c}41 \\
11 \\
14 \\
3\end{array}$ & $\begin{array}{r}59.4 \\
15.9 \\
20.3 \\
4.4\end{array}$ \\
\hline $\begin{array}{l}\text { Infección genitourinaria en la madre } \\
\text { Ninguna } \\
\text { ITU } \\
\text { Vulvovaginosis } \\
\quad \text { Estreptococo agalactiae }\end{array}$ & $\begin{array}{c}31 \\
7 \\
5 \\
4\end{array}$ & $\begin{array}{r}44.9 \\
10.1 \\
7.3 \\
5.8\end{array}$ \\
\hline $\begin{array}{l}\text { Complicaciones periparto } \\
\text { Ninguna } \\
\text { RPMO } \\
\text { SPP } \\
\text { THAE }\end{array}$ & $\begin{array}{c}44 \\
16 \\
6 \\
4\end{array}$ & $\begin{array}{r}58.7 \\
21.3 \\
8.0 \\
5.3\end{array}$ \\
\hline $\begin{array}{l}\text { Pacientes con CN sin y con enfermedad } \\
\text { sistémica } \\
\text { Sin enfermedad sistémica (neonatos con } \\
\text { CN solamente) } \\
\text { Con enfermedad sistémica asociada }\end{array}$ & 21 & 30.4 \\
\hline
\end{tabular}

*Promedio \pm desviación estándar.

CN: conjuntivitis neonatal; ITU: infección del tracto urinario; RPMO: ruptura prematura de membranas ovulares; SPP: síndrome de parto pretermino; THAE. trastorno hipertensivo asociado al embarazo; UCEN: unidad de cuidados especiales neonatales; UCIN: unidad de cuidados intensivos neonatales.

Al realizar este estudio en un tercer nivel de complejidad, un importante número de RN presentaban una enfermedad sistémica además de la $\mathrm{CN}$ ( $\mathrm{n}=40$, $69.6 \%$ ). De estas enfermedades sistémicas, la sepsis ( $n=18,23 \%)$, seguida de bronquiolitis $(n=14,18 \%)$ y la neumonía $(n=10,13 \%)$ fueron las más frecuentes. En el $30.4 \%$ ( $n=21)$ no se encontró ningún tipo de infección sistémica. Registrar la presencia de enfermedad sistémica fue relevante para determinar el tipo de terapia antibiótica sistémica recibida en estos pacientes, comparado con quienes solo presentaban $\mathrm{CN}$. Esto se discutirá más adelante en tratamiento (Tabla 2). 


\section{Hallazgos microbiológicos}

Los microorganismos encontrados con mayor frecuencia fueron: Staphylococcus epidermidis $(n=20$, $28.99 \%$ ), seguido de Staphylococcus aureus $(n=12$, $17.39 \%)$ y Haemophilus influenzae ( $n=9,13.04 \%)$. Con las pruebas realizadas no se obtuvo crecimiento de $N$. gonorrhoeae, ni hubo resultado positivo para C. trachomatis. En ocho casos (11.54\%) se encontró una infección polimicrobiana. A todos los pacientes se les realizó agar sangre y agar chocolate $(n=69)$, al $90 \%$ de los pacientes $(n=62)$, antígeno para $C$. thacomatis, y al $60 \%(n=41)$, Thayer-Martin. En la tabla 3 se muestra la totalidad de los microorganismos aislados.

Al evaluar el tipo de microorganismo aislado de acuerdo con la infección periparto reportada en la madre, se encontró que la infección del tracto urinario, seguida de la vulvovaginosis, fue la infección más frecuente en las madres de los neonatos con $\mathrm{CN}$ por $S$. epidermidis y $S$. aureus. También se evaluaron las complicaciones periparto en relación con estos microorganismo, y se encontró la RPMO y el SPP como las principales. Estos fueron los factores de riesgo detectados con mayor frecuencia, y, aunque no puede establecerse una relación causal, sí podría considerarse como un factor predisponente (Tabla 4).

\section{Terapia antibiótica tópica y sistémica}

La antibioticoterapia sistémica más utilizada fue la ampicilina. Se usó en el $29 \%$ de los casos $(n=29)$, seguida de la amikacina $(n=18,18 \%)$ y la ampicilina/ amikacina ( $n=14,14 \%)$. El antibiótico tópico más empleado fue la sulfacetamida en el $67 \%$ de los casos $(n=46)$, la gentamicina en el $23 \%(n=16)$ de los casos y el ciprofloxacino en el $4 \%$ de los casos $(n=3)$. Estos resultados reportados fueron en pacientes que tenían una enfermedad sistémica concomitante con la $\mathrm{CN}$. Asimismo, se relacionó el tratamiento sistémico utilizado en los pacientes con $\mathrm{CN}$ sin presencia de enfermedad sistémica, y la combinación de azitromicina y ceftriaxona fue la más empleada $(33 \%, n=7)$. En los pacientes sin enfermedad sistémica asociada, la sulfacetamida sódica también fue el antibiótico tópico más usado (Tablas 5,6 y 7).

El perfil de resistencia antibiótica se analizó para todos los microorganismos aislados. La mayoría de microorganismos no tenían resistencia antibiótica. Sin embargo, para la familia de Staphylococcus se encontró resistencia a la oxacilina en el $26 \%(n=18)$, y a las
Tabla 2. Enfermedad sistémica asociada en el neonato $(n=69)^{*}$

\begin{tabular}{|l|c|c|}
\hline Enfermedad sistémica en el neonato & n & $\%$ \\
\hline Ninguna & 21 & 30.4 \\
\hline Sepsis & 18 & 25 \\
\hline Bronquiolitis & 14 & 19 \\
\hline Neumonía & 10 & 13.3 \\
\hline Distrés respiratorio & 7 & 9.7 \\
\hline Meningoencefalitis & 1 & 1.3 \\
\hline Otitis & 1 & 1.3 \\
\hline Total & 72 & 100 \\
\hline
\end{tabular}

*Un paciente podía tener más de una enfermedad sistémica asociada.

Tabla 3. Frecuencia de microorganismos aislados en muestras de secreción conjuntival de neonatos

\begin{tabular}{|l|l|l|}
\hline Microorganismo & n & $\%$ \\
\hline Staphylococcus epidermidis & 20 & 28.99 \\
\hline Staphylococcus aureus & 12 & 17.39 \\
\hline Haemophilus influenzae & 9 & 13.04 \\
\hline Staphylococcus hominis & 5 & 7.25 \\
\hline Escherichia coli & 4 & 5.80 \\
\hline Serratia marcescens & 4 & 5.80 \\
\hline Staphylococcus haemolyticus & 3 & 4.35 \\
\hline Streptococcus pneumoniae & 3 & 4.35 \\
\hline Klebsiella pneumoniae & 2 & 2.90 \\
\hline Enterobacter cloacae & 1 & 1.45 \\
\hline Klebsiella oxytoca & 1 & 1.45 \\
\hline Staphylococcus coagulasa negativo & 1 & 1.45 \\
\hline Staphylococcus sciuri & 1 & 1.45 \\
\hline Staphylococcus warneri & 1 & 1.45 \\
\hline Stenotrophomonas maltophila & 1 & 1.45 \\
\hline Streptococcus vestibularis & 1 & 1.45 \\
\hline Chlamydia trachomatis & 0 & 0 \\
\hline Neisseria gonorrhoeae & 09 & 0 \\
\hline Total & 100 \\
\hline
\end{tabular}

tetraciclinas en el $15 \%(n=11)$, lo que indica un importante número de pacientes con Staphylococcus aureus meticilinorresistente. 
Tabla 4. Microorganismos relacionados con factores de riesgo periparto

\begin{tabular}{|c|c|}
\hline Infección materna & Microorganismos aislados \\
\hline Ninguna $(n=31)$ & $\begin{array}{l}\text { Staphylococcus epidermidis }(\mathrm{n}=9) \\
\text { Staphylococcus aureus }(\mathrm{n}=5)\end{array}$ \\
\hline $\begin{array}{l}\text { Infección urinaria } \\
(\mathrm{n}=7)\end{array}$ & Staphylococcus epidermidis $(\mathrm{n}=2)$ \\
\hline Vulvovaginosis $(n=5)$ & Staphylococcus epidermidis $(\mathrm{n}=1)$ \\
\hline $\begin{array}{l}\text { Estreptococo } \\
\text { agalactiae }(n=4)\end{array}$ & Staphylococcus epidermidis $(\mathrm{n}=1)$ \\
\hline \multicolumn{2}{|c|}{ Complicación materna no infecciosa } \\
\hline Ninguna $(n=42)$ & $\begin{array}{l}\text { Staphylococcus epidermidis }(\mathrm{n}=17) \\
\text { Haemophilus influenzae }(\mathrm{n}=6)\end{array}$ \\
\hline $\begin{array}{l}\text { Ruptura prematura } \\
\text { membranas }(n=16)\end{array}$ & $\begin{array}{l}\text { Staphylococcus epidermidis }(\mathrm{n}=3) \\
\text { Staphylococcus aureus }(\mathrm{n}=3)\end{array}$ \\
\hline $\begin{array}{l}\text { Síndrome parto } \\
\text { prematuro }(n=6)\end{array}$ & $\begin{array}{l}\text { Staphylococcus aureus }(\mathrm{n}=2) \\
\text { Staphylococcus hominis }(\mathrm{n}=2)\end{array}$ \\
\hline $\begin{array}{l}\text { Trastorno hipertensivo } \\
(n=4)\end{array}$ & Staphylococcus aureus $(\mathrm{n}=3)$ \\
\hline \multicolumn{2}{|l|}{ Infección neonatal } \\
\hline Ninguna $(n=27)$ & $\begin{array}{l}\text { Staphylococcus epidermidis }(\mathrm{n}=11) \\
\text { Escherichia coli }(\mathrm{n}=3)\end{array}$ \\
\hline Sepsis $(n=14)$ & $\begin{array}{l}\text { Staphylococcus aureus }(n=5) \\
\text { Staphylococcus epidermidis }(n=4)\end{array}$ \\
\hline Bronquiolitis ( $n=18$ ) & $\begin{array}{l}\text { Haemophilus influenzae }(n=8) \\
\text { Staphylococcus aureus }(n=4)\end{array}$ \\
\hline Neumonía $(n=10)$ & $\begin{array}{l}\text { Staphylococcus epidermidis }(\mathrm{n}=3) \\
\text { Haemophilus influenzae }(\mathrm{n}=2)\end{array}$ \\
\hline
\end{tabular}

\section{Discusión}

La CN es la inflamación más común de la conjuntiva en el neonato. Está causada en la mayoría de los casos por agentes infecciosos y representa una de las principales causas de ceguera prevenible en el primer mes de vida ${ }^{15,16}$, especialmente en países en desarrollo, con deficiencias en la calidad de la atención materna y del neonato, por lo que claramente sigue siendo una condición grave y de interés ${ }^{17}$ para este grupo de población. El tipo de microorganismo, riesgo de infección y prevalencia varían con la zona geográfica, condiciones del parto y manejo profiláctico tópico aplicado ${ }^{18,19}$.

En la década de 1970, la CN era causada principalmente por microorganismos asociados a infecciones de trasmisión sexual adquiridos en el canal del parto ${ }^{16,17}$ : N. gonorrhoeae era reportada hasta en un $50 \%$ de los $\operatorname{casos}^{21,22}, y$, en segundo lugar, $C$. trachomatis. En un estudio realizado en Corea del Sur, reportaron una prevalencia acumulada de la $\mathrm{CN}$ gonocócica en
Tabla 5. Terapia antibiótica sistémica utilizada en conjuntivitis neonatal $(n=69)^{*}$

\begin{tabular}{|l|l|l|}
\hline Tratamiento sistémico & Total & $\%$ \\
\hline Ampicilina IV & 29 & 29 \\
\hline Amikacina IV & 18 & 18 \\
\hline Ampicilina/amikacina IV & 14 & 14 \\
\hline Azitromicina IV & 8 & 8 \\
\hline Piperacilina/tazobactam IV & 7 & 7 \\
\hline Ceftriaxona IV & 7 & 7 \\
\hline Ampicilina/gentamicina IV & 7 & 7 \\
\hline Claritromicina IV & 2 & 2 \\
\hline Vancomicina IV & 2 & 2 \\
\hline Cefotaxima IV & 2 & 2 \\
\hline Ceftriaxona IM & 1 & 1 \\
\hline Ampicilina/cefepima IV & 1 & 1 \\
\hline Oximetazolina IV & 1 & 1 \\
\hline Cefazolina IV & 1 & 1 \\
\hline Claritromicina IV & 1 & 1 \\
\hline Total & 101 & 100 \\
\hline Vn & & 7 \\
\hline
\end{tabular}

*Un paciente pudo recibir más de un antibiótico IV. IM: intramuscular; IV: intravenoso.

descenso, del $25 \%$ en 1982 , del $9 \%$ en 1991 y del $0.9 \%$ en $1997^{23}$.

En cuanto a clamidia, si bien ha disminuido su prevalencia, no ha sido paralela a la del gonococo ${ }^{24}$. Un estudio realizado a nivel local en $1997^{25}$ reportó la etiología de la conjuntivitis neonatal en 102 niños. Obtuvieron aislamientos de C. trachomatis en el 33\% de los casos, $H$. influenzae en el $18 \%$ y $S$. aureus en el $15 \%$. Analizando estos resultados se puede considerar que, si bien $C$. trachomatis fue la primera causa de $\mathrm{CN}$ en los últimos años del siglo XX, los microorganismos de la piel y vías respiratorias (no gonocócicos) ya empezaban a cobrar un lugar importante.

La mayoría de los estudios realizados en las últimas décadas coinciden en que ha habido un cambio en la etiología microbiana de la $\mathrm{CN}^{10,11,15,18,24-27}$, pasando de microorganismos adquiridos en el canal del parto (N. gonorrhoeae y C. trachomatis) a microorganismos de la piel y de las vías respiratorias superiores ${ }^{10,28}$.

Los agentes encontrados con mayor frecuencia en este estudio fueron de origen bacteriano no gonocócico: S. epidermidis ( $28.9 \%$ de los casos), S. aureus (17.39\% de los casos) y $H$. influenzae $(13.04 \%$ de los casos). Si bien se ha considerado al $S$. epidermidis 
Tabla 6. Terapia antibiótica sistémica utilizada en conjuntivitis neonatal sin enfermedad sistémica $(n=21)$

\begin{tabular}{|l|c|c|}
\hline Tratamiento sistémico & Total & $\%$ \\
\hline Azitromicina/ceftriaxona & 7 & 33 \\
\hline Amikacina & 4 & 19 \\
\hline Ampicilina/amikacina & 3 & 14 \\
\hline Ampicilina & 2 & 10 \\
\hline Cefotaxima & 2 & 10 \\
\hline Piperacilina/tazobactam & 2 & 10 \\
\hline Ceftriaxona & 1 & 4 \\
\hline Total & 21 & 100 \\
\hline
\end{tabular}

Tabla 7. Terapia antibiótica tópica utilizada en conjuntivitis neonatal $(\mathrm{n}=69)$

\begin{tabular}{|l|c|c|}
\hline Tratamiento ocular & Total & $\%$ \\
\hline Sulfacetamida & 46 & 67 \\
\hline Gentamicina & 16 & 23 \\
\hline Ciprofloxacino & 3 & 4 \\
\hline Tobramicina & 2 & 3 \\
\hline Moxifloxacino & 2 & 3 \\
\hline Total & 69 & 100 \\
\hline
\end{tabular}

como flora saprofítica y su papel patogéno no es del todo claro, se plantea que la combinación de condiciones específicas del neonato (su estructura epidérmica, un sistema inmune neonatal aún inmaduro y el uso inapropiado de antibióticos) podría convertir en patógenas a bacterias menos virulentas ${ }^{29}$.

En otro estudio más reciente, desarrollado también en Corea del Sur y publicado en el año 2017, no obtuvieron ningún caso de infección por $C$. trachomatis ni $N$. gonorrhoea y los principales agentes causales aislados pertenecían a la famila de Staphylococcus sp. ${ }^{10}$. Estos resultados ${ }^{18}$ son muy similares a los obtenidos en nuestro estudio, donde predominaron las bacterias no gonocócicas, y no identificamos ningún aislamiento positivo para $N$. gonorrhoeae ni para C. trachomatis.

En más del $50 \%$ de los casos no se encontró un factor de riesgo claramente relacionado con una mayor prevalencia de estos microorganismos, sin embargo, la ITU fue la infección periparto más frecuente. No fue reportada ninguna materna con infección vaginal por gonococo ni clamidia, lo que podría indicar que en nuestro medio existen estándares adecuados en la atención prenatal de la materna, similares a los descritos en países desarrollados ${ }^{30}$.

Los factores de riesgo relacionados con la aparición de $C N$ bacteriana por $S$. epidermidis y $S$. aureus fueron la ITU, la RPMO y el SPP. Diferentes estudios han mostrado resultados similares, lo que significa que estos factores de riesgo deben ser prevenidos, identificados y tratados ${ }^{5,31}$. Sin embargo, no se identificó ningún factor de riesgo en más del $50 \%$ de los casos (Tabla 1).

La sulfacetamida y la gentamicina fueron los antibióticos tópicos más empleados. A nivel sistémico fue la ampicilina y la amikacina, sin embargo, la mayoría de estos pacientes tenían una enfermedad sistémica asociada (sepsis), por tanto, no es posible determinar si el antibiótico sistémico fue usado preferiblemente por la enfermedad de base del paciente o la presencia de $\mathrm{CN}$. Por esta razón, se hizo un análisis de pacientes sin enfermedad sistémica con $\mathrm{CN}$. En este grupo de pacientes, la azitromicina y la ceftriaxona fueron los antibióticos sistémicos más utilizados. Esto demuestra que todavía se continúan utilizando antibióticos de muy amplio espectro que abarquen microorganismos más agresivos (C. trachomatis y N. gonorrhoeae), aunque hoy en día sean los menos frecuentes, lo que aumenta el riesgo de desarrollar resistencia antibiótica.

Por los resultados obtenidos en este estudio se podría considerar que para los casos de CN sin infección sistémica asociada, los antibióticos tópicos tradicionales, como gentamicina, tobramicina y sulfacetamida sódica, son una opción apropiada, puesto que se ha demostrado que estos microorganismos de la piel y del ambiente tienen un comportamiento menos agresivo en el ojo y mínimo riesgo de producir complicaciones sistémicas y locales ${ }^{5,10}$.

Para el grupo de Staphylococcus, responsables de cerca del $50 \%$ de la $\mathrm{CN}$ en nuestro estudio, hubo una resistencia a la oxacilina del $26 \%$ y a las tetraciclinas del $15 \%$, menor a la reportada en otras latitudes - hasta del $83.7 \%$ a la meticilina- ${ }^{10}$.

Nuestro estudio tiene varias limitaciones. En primer lugar, los cultivos especiales de Tayer-Marthyn para $N$. gonorrhoeae y pruebas específicas para C. trachomatis no se realizaron en todos los pacientes, lo que puede asociarse con un sesgo en los resultados. La evaluación prospectiva entre marzo y noviembre de 2018 pretendió reducir este sesgo. Adicionalmente, el desarrollo del estudio en un único centro de muy alta complejidad limita su validez externa. Sin embargo, se obtuvieron hallazgos similares a otras regiones del mundo, lo que disminuye esta posibilidad. 


\section{Conclusión}

El perfil epidemiológico de la $\mathrm{CN}$ ha cambiado, no solo en nuestro medio sino a nivel mundial. Esto lleva a enfrentarnos a otro tipo de microorganismos no gonocócicos (S. epidermidis y $S$. aureus) que por fortuna no se asocian con complicaciones graves como sí se observan con microorganismos gonocócicos. La principal vía de infección en tiempos recientes se considera que es la piel y la vía respiratoria, mientras que en el pasado fue el canal del parto. Por esto, la prevalencia de la $\mathrm{CN}$ debe disminuir con el lavado adecuado de manos antes de entrar en contacto con los neonatos.

Por el momento, la recomendación es usar los antibióticos tópicos tradicionales, como la tobramicina y la sulfacetamida, para los casos de CN sin enfermedad sistémica asociada, y para los casos de $\mathrm{CN}$ con infeccion sistémica, la elección del antibiótico sistémico dependerá del tipo de infección concomitante. A pesar de la emergencia de microorganismos comensales de la piel y del ambiente, para todos los casos se deben solicitar cultivos de bacterias aerobias, $N$. gonorrhoeae y $C$. trachomatis, y orientar el tratamiento según el aislamiento.

La disminución de la prevalencia de microorganismos gonocócicos y de la clamidia en la $\mathrm{CN}$ se ha logrado con el tamizaje de infecciones en la madre, la identificación de factores de riesgo (como la ITU, la RPMO y el SPP), mejoras en la calidad de la atención prenatal y profilaxis antibiótica en el neonato. Todas estas medidas han cambiado el panorama de la CN disminuyendo los índices de ceguera que fueron en una época bastante altos.

\section{Conflicto de intereses}

Todos los autores declaran la inexistencia de conflicto de intereses.

\section{Responsabilidades éticas}

Protección de personas y animales. Los autores declaran que para esta investigación no se han realizado experimentos en seres humanos ni en animales.

Confidencialidad de los datos. Los autores declaran que han seguido los protocolos de su centro de trabajo sobre la publicación de datos de pacientes.

Derecho a la privacidad y consentimiento informado. No se requirió recolección de consentimiento informado de los pacientes ya que el estudio se clasificó sin riesgo, con obtención de la información de fuentes secundarias.

\section{Bibliografía}

1. Hammerschlag MR. Chlamydial and Gonococcal Infections in Infants and Children. Clin Infect Dis. 2011;53(suppl_3):S99-102.

2. Shahid E, Shaikh A, Aziz S, Rehman A. Frequency of Ocular Diseases in Infants at a Tertiary Care Hospital. Korean J Ophthalmol KJO. 2019;33(3):287-93.

3. Gilbert C, Foster A. Childhood blindness in the context of VISION 2020-the right to sight. Bull World Health Organ. 2001;79(3):227-32.

4. Cortés VR, Hernández R. Enfermedad infecciosa neonatal y gérmenes patógenos en un hospital de tercer nivel. (Spanish). Bol Med Hosp Infant Mex. 2006;63:S33-4

5. Mohammed A, Ibrahim M, Mustafa A, Ihesiulor U. Maternal risk factors for neonatal conjunctivitis in Aminu Kano Teaching Hospital, Kano, Nigeria. Niger J Basic Clin Sci. 2013;10(2):60.

6. Krohn MA, Hillier SL, Bell TA, Kronmal RA, Grayston JT. The Bacterial Etiology of Conjunctivitis in Early Infancy. Am J Epidemiol. 1993;138(5):326-32.

7. Palafox SKV, Jasper S, Tauber, Allyson D, Foster SC. Ophthalmia Neonatorum. J Clin Exp Ophthalmol [Internet]. 2010;2:119el 27 de diciembre de 2010 [citado el 28 de diciembre de 2017];2(1). Disponible en: https://www. omicsonline.org/ophthalmia-neonatorum-2155-9570.1000119.php?aid=1094

8. Zikic A, Schünemann H, Wi T, Lincetto O, Broutet N, Santesso N. Treatment of Neonatal Chlamydial Conjunctivitis: A Systematic Review and Meta-analysis. J Pediatr Infect Dis Soc. 2018;7(3):e107-15.

9. Afjeiee SA, Tabatabaei SR, Fallah F, Shiva F, Zanjani NT, Fard AT, et al. A microbiological study of neonatal conjunctivitis in two hospitals in Tehran, Iran. Asian Pac J Trop Dis. 2013;3(6):429-33.

10. Pak KY, Kim SI, Lee JS. Neonatal Bacterial Conjunctivitis in Korea in the $21^{\text {st }}$ Century. Cornea. 2017;36(4):415-8.

11. Alexandre I, Cortes N, Justel M, Fernández I, Ortíz de Lejarazu R, Pastor JC. The value of simple microbiological studies for on-site screening of acute neonatal conjunctivitis in Angola. J Ophthalmic Inflamm Infect. 2014:4(1):1.

12. Kara M, Kıvanç SA, Olcaysü OO, Akova Budak B, Özmen AT, Kıvanç M, et al. The newborn conjunctival flora at the post delivery 24 hours. J Curr Ophthalmol. 2018:30(4):348-52.

13. Rodríguez Gazquez M de los Á, Correa Muñoz E, Ospina Ospina S, Giraldo Jiménez C. Etiología bacteriana de la conjuntivitis neonatal. Metrosalud. Medellín, 1994. Colombia Medica. 1997;28(2):58-61.

14. Trujillo H, Restrepo C, Correa A, Robledo C, Harry N, Mejía GI. Conjuntivitis por Chlamydia trachomatis en recién nacidos y lactantes. Med UPB. 1989;8(1):27-32.

15. Schaller UC, Klauss V. Is Credé's prophylaxis for ophthalmia neonatorum still valid? Bull World Health Organ. 2001;79(3):262-3.

16. Mallika P, Asok T, Faisal H, Aziz S, Tan A, Intan G. Neonatal Conjunctivitis - a Review. Malays Fam Physician Off J Acad Fam Physicians Malays. 2008;3(2):77-81.

17. Gul SS, Jamal M, Khan N. Ophthalmia neonatorum. J Coll Physicians Surg--Pak JCPSP. 2010;20(9):595-8.

18. McAnena L, Knowles SJ, Curry A Cassidy L. Prevalence of gonococcal conjunctivitis in adults and neonates. Eye Lond Engl. julio de 2015;29(7):875-80.

19. Nsanze H, Dawodu A, Usmani A, Sabarinathan K, Varady E. Ophthalmia neonatorum in the United Arab Emirates. Ann Trop Paediatr. 1996; 16(1):27-32

20. Nishida H, Risemberg HM. Silver nitrate ophthalmic solution and chemical conjunctivitis. Pediatrics. 1975;56(3):368-73.

21. Armstrong $\mathrm{JH}$, Zacarias F, Rein MF. Ophthalmia neonatorum: a chart review. Pediatrics. 1976;57(6):884-92.

22. Laga M, Nzanze H, Brunham R, Maitha G, D'Costa L, Mati JK, et al. Epidemiology of ophthalmia neonatorum in Kenya. The Lancet. 1986; 328(8516):1145-9.

23. Kim BL, Choi TH, Lee HB. The Neonatal Conjunctivitis. J Korean Ophthalmol Soc. 1997;38(9):1537-44.

24. Di SB, Mirta DH, Janer M, Rodríguez MF, Sauka D, Magariños F, et al. Incidence of Chlamydia trachomatis and other potential pathogens in neonatal conjunctivitis. Int J Infect Dis IJID Off Publ Int Soc Infect Dis. 2001:5(3):139-43.

25. Rodríguez Gazquez M de los Á, Correa Muñoz E, Ospina Ospina S, Giraldo Jiménez C. Etiología bacteriana de la conjuntivitis neonatal. Colomb Médica [Internet]. 1997 [citado el 13 de noviembre de 2017];28(2). Disponible en: http://www.redalyc.org/resumen.oa?id=28328202.

26. Honkila M, Renko M, Ikäheimo I, Pokka T, Uhari M, Tapiainen T. Aetiology of neonatal conjunctivitis evaluated in a population-based setting. Acta Paediatr Oslo Nor 1992. 2018;107(5):774-9.

27. Kokou V, Nidain M, Kassoula NB, Kwassi F-A, Meba B, Patrice BK. [Neonatal conjunctivitis in the district of Glidji in Southern Togo: a cross sectional study about 159 new-borns]. Pan Afr Med J. 2016;24:42.

28. Satpathy G, Behera HS, Ahmed NH. Chlamydial eye infections: Current perspectives. Indian J Ophthalmol. 2017;65(2):97-102.

29. Tang S, Li M, Chen H, Ping G, Zhang C, Wang S. A chronological study of the bacterial pathogen changes in acute neonatal bacterial conjunctivitis in southern China. BMC Ophthalmol. 2017;17(1):174

30. Foster A, Klauss V. Ophthalmia neonatorum in developing countries. N Engl J Med. 1995;332(9):600-1.

31. Isenberg SJ, Apt L, Wood M. The influence of perinatal infective factors on ophthalmia neonatorum. J Pediatr Ophthalmol Strabismus. 1996;33(3):185-8. 